\title{
EXOTIC CLASSES FOR MEASURED FOLIATIONS
}

\author{
BY STEVEN HURDER
}

A measured foliation $(F, \mu)$ is a $C^{2}$-foliation $F$ on a smooth manifold $M$ and a transverse invariant measure $\mu$ for $F$ [14]. Inspired by the foliation index theorem of Connes $[4,5]$, we study the result of integrating normal data to $F$ over the leaf space $M / F$. This produces new secondary-type exotic classes for measured foliations [7]. These classes have applications to $S L_{q}$-foliations, to the study of groups of volume-preserving diffeomorphisms, and also are useful for relating the geometry of $F$ to the values of the usual secondary classes $[8,9]$.

THEOREM 1. Let $(F, \mu)$ be a measured foliation of codimension $q$ on $M$. If either $M$ is closed and orientable, or $\mu$ is absolutely continuous (so it is represented by a closed form $d \mu$ ), then there is a well-defined characteristic map

$$
\chi_{\mu}: H^{*}\left(\mathrm{gl}_{q}, O_{q}\right) \rightarrow H^{*+q}(M) .
$$

We call the image of $\chi_{\mu}$ the $\mu$-classes of $(F, \mu)$.

For $M^{m}$ compact and $y_{I} \in H^{n}\left(\mathrm{gl}_{q}, O_{q}\right)$, the class $\chi_{\mu}\left(y_{I}\right)$ is defined as the geometric current in $H_{m-n-q}(M)$ obtained by integrating over the leaf space of $F$, via $\mu$, the leaf classes corresponding to $y_{I}$. Duality then produces the invariant in $H^{n+q}(M)$. If $d \mu$ is a closed form representing $\mu$, then a cocycle representing $\chi_{\mu}\left(y_{I}\right)$ is $\Delta\left(y_{I}\right) \cdot d \mu$, where $\Delta: W O_{q} \rightarrow A^{\circ}(M)$ is the secondary map for $F,[2,10]$. Complete details and properties of $\chi_{\mu}$ are described in [7] .

The values of the $\mu$-classes depend on the measure $\mu$ and the dynamical behavior of $F$ in a neighborhood of the support of $\mu$. It is conjectured that subexponential growth of the leaves of $F$ implies the $\mu$-classes vanish; this can be shown in some cases. Examples can be constructed for which all of the $\mu$-classes are nontrivial.

The canonical measure associated to an $S L_{q}$-foliation $(F, \omega)$-where $\omega$ is a transverse invariant volume form-defines a characteristic map $\chi_{\omega}: H^{*}\left(\mathrm{sl}_{q}, S O_{q}\right)$ $\rightarrow H^{*+q}(M)$, and these come from universal classes for the Haefliger classifying space $B \Gamma_{S L_{q}}$. There are additional $\mu$-classes for measured foliations with framed normal bundles, and corresponding universal classes for $B \bar{\Gamma}_{S L_{q}}$, the homotopy fiber of $B \Gamma_{S L_{q}} \rightarrow B S L_{q}$.

Received by the editors February 23, 1982.

1980 Mathematics Subject Classification. Primary 57R30; Secondary 57R20, 28D15. 
THEOREM 2. The characteristic maps

$$
\begin{aligned}
& \chi: H^{n}\left(\mathrm{sl}_{q}, S O_{q}\right) \rightarrow H^{n+q}\left(B \Gamma_{S L_{q}}\right), \\
& \chi s: H^{n}\left(\mathrm{sl}_{q}\right) \rightarrow H^{n+q}\left(B \bar{\Gamma}_{S L_{q}}\right)
\end{aligned}
$$

are injective for $n<[(q-1) / 4]$. Further, $\chi^{s}$ is injective on the image $H^{*}\left(\mathrm{so}_{q}\right)$ $\rightarrow H^{*}\left(\mathrm{sl}_{q}\right)$.

To show nontriviality, we compute the values of the $\mu$-classes for the foliations obtained by suspending the action of $S L_{q} Z$ on $T^{q}$. This type of example was suggested to us by $\mathrm{W}$. Thurston. The corresponding characteristic map is related to the Van Est map of continuous cohomology $H^{*}\left(\mathrm{sl}_{q}, S \mathrm{SO}_{q}\right) \rightarrow$ $H^{*}\left(S L_{q} Z ; \mathbf{R}\right)$, which is injective in degrees less than [(q-1)/4] by Borel [1] Note that $\chi^{s}$ is nontrivial for all $q \geqslant 3$, but Theorem 2 only asserts $\chi$ is nontrivial for $q \geqslant 25$. We do not know of $S L_{q}$-foliations with small codimension and nontrivial $\mu$-classes from $\chi$.

Let $\omega$ be a volume form on $\mathbf{R}^{q}$ with infinite total mass and $\operatorname{Diff}_{\omega}^{c} \mathbf{R}^{q}$ the group of compactly supported diffeomorphisms which preserve $\omega$. We use McDuff's generalization of the Mather-Thurston theorem [11] and Theorem 2 to prove

COROLLARY 1.. There are inclusions of $\mathbf{Q}$-vector spaces

$$
H_{n}\left(\mathrm{so}_{q} ; \mathbf{R}\right) \hookrightarrow H_{n}\left(B \overline{\operatorname{Diff}_{\omega}^{c}} \mathbf{R}^{q} ; \mathbf{Q}\right)
$$

for $n<q$, and $\mathbf{R} \subseteq H_{3}\left(B \overline{\operatorname{Diff}_{\omega}^{c}} \mathbf{R}^{q} ; \mathbf{Q}\right)$ for all $q \geqslant 3$.

It was shown that $H_{1}\left(B \overline{\operatorname{Diff}_{\omega}^{c}} \mathbf{R}^{q} ; Z\right)=0$ for $q>2$ by Thurston-Banyaga. Corollary 1 gives the first nonvanishing results for the group homology in degrees less than $q+1$; in degrees $\geqslant q+1$, the secondary classes of $S L_{q}$-foliations detect nontrivial homology of $B \overline{\operatorname{Diff}_{\omega}^{c}} R^{q}$. McDuff has investigated in [12] the geometrical significance of some of these new invariants for $\operatorname{Diff}_{\omega}^{c} \mathbf{R}^{q}$ and also defined further interesting classes.

The residuable secondary classes are the cocycles $y_{I} c_{J}$ in $H^{*}\left(W O_{q}\right)$ with degree $c_{J}=2 q$ maximal. The "integration over the fiber" process is faithful on these classes, so a residue theory can be developed for them. Given a measured foliation $(F, \mu)$ with support $\mu=M$, the residuable classes decompose into the measure class $d \mu$ product with a leaf invariant. This observation can be used to relate the residuable secondary classes with the geometry of $F$.

THEOREM 3. Let $F$ be a codimension q compact foliation (that is, each leaf of $F$ is compact) on a closed manifold $M$. Each residuable secondary class $\Delta_{*}\left(y_{I} c_{J}\right) \in H^{*}(M)$ is then zero. 
The idea of the proof is to integrate $\Delta\left(y_{I} c_{J}\right)$ over $M$, decompose this integral over saturated sets-the Epstein filtration of the bad set-where each saturated set has a transverse invariant measure of maximal support. Each integral decomposes into a weighted sum of leaf classes, and then we show the leaf classes for a compact foliation uniformly vanish. Details appear in [8].

For codimension one foliations remarkable progress has been made in relating the geometry of a foliation with its Godbillon-Vey invariant $[3,13]$. For higher codimensions, it is expected that a geometric interpretation of the residuable secondary classes can be achieved by utilizing the techniques of the proof of Theorem 3, the residue theorem for foliations [6] and the properties of the $\mu$-classes. Some progress on this problem is given in [9] .

ADDED IN PROOF. G. Duminy has recently proved that a codimension-one foliation on a compact manifold with nonvanishing Godbillon-Vey invariant must have a resilient leaf ( $L$ 'Invariant de Godbillon-Vey d'un feuilletage se localise dans les feuilles ressort, preprint.)

\section{REFERENCES}

1. A. Borel, Stable real cohomology of arithmetic groups, Ann. Sci. École Norm. Sup. 4e 7 (1974), 235-272.

2. R. Bott and A. Haefliger, On characteristic classes of $\Gamma$-foliations, Bull. Amer. Math. Soc. 78 (1972), 1039-1044.

3. J. Cantwell and L. Conlon, A vanishing theorem for the Godbillon-Vey invariant of foliated manifolds, to appear (1981).

4. A. Connes, Sur la théorie non-commutative de l'integration, Lecture Notes in Math., vol. 725, Springer-Verlag, Berlin and New York, 1979, pp. 19-143.

5. A. Connes and G. Skandalis, Théorème de l'indice pour les feuilletages, C. R. Acad. Sci. Paris Sér. A 292 (1981), 871-876.

6. J. Heitsch, Flat bundles and residues for foliations, to appear (1981).

7. S. Hurder, Global invariants for measured foliations, to appear (1981).

8. - Vanishing of secondary classes for compact foliations, to appear (1982). (1982).

9. - Growth of leaves and differential invariants of foliations, in preparation

10. F. Kamber and P. Tondeur, Foliated bundles and characteristic classes, Lecture Notes in Math., vol. 493, Springer-Verlag, Berlin and New York, 1975, pp. 1-294.

11. D. McDuff, The homology of groups of volume preserving diffeomorphisms, Ann. École Norm. Sup. (to appear).

12. Some canonical cohomology classes on groups of volume preserving diffeomorphisms, Trans. Amer. Math. Soc. (to appear).

13. T. Mizutani, S. Morita and T. Tsuboi, The Godbillon-Vey classes of codimension one foliations which are almost without holonomy, Ann. of Math. (2) 113 (1981), 515-527.

14. J. Plante, Foliations with measure preserving holonomy, Ann. of Math. (2) 102 (1975), 327-361.

DEPARTMENT OF MATHEMATICS, PRINCETON UNIVERSITY, PRINCETON, NEW JERSEY 08544 
